\title{
Dynamic changes in transposable element and gene methylation in mulberry (Morus notabilis) in response to Botrytis cinerea
}

\author{
Youchao Xin (1) ${ }^{1,2}$, Bi Ma (1) , Qiwei Zeng ${ }^{1}$, Wenmin $\mathrm{He}^{1}$, Meiling Qin and Ningjia $\mathrm{He}^{1}$
}

\begin{abstract}
DNA methylation has been proposed to regulate plant stress resistance. However, the dynamic changes in DNA methylation in woody plants and their correlations with pathogenic responses are not fully understood. Here, we present single-base maps of the DNA methylomes of mulberry (Morus notabilis) leaves that were subjected to a mock treatment or inoculation with Botrytis cinerea. Compared with the former, the latter showed decreased mCG and $\mathrm{mCHG}$ levels and increased $\mathrm{mCHH}$ levels. DNA methylation inhibitors reduced resistance gene methylation levels and enhanced mulberry resistance, suggesting that the hypomethylation of resistance genes affects mulberry resistance to B. cinerea. Virus-induced gene silencing of MnMET1 enhanced the expression of mulberry-resistance genes, thereby increasing the plant's resistance to B. cinerea. We also found that MITEs play a dominant role in controlling DNA methylation levels. MITEs appear to be the main sources of 24-nt siRNAs that regulate gene expression through the RNA-directed DNA methylation pathway.
\end{abstract}

\section{Introduction}

Botrytis cinerea is the second most common plant fungal pathogen. It is a necrotizing pathogen that destroys host cells with a series of toxic molecules and through the plant's own defense mechanisms. This fungus infects more than 200 dicotyledonous plants, as well as some monocots ${ }^{1}$. It infects cash crops, such as tomatoes and petunias, and causes approximately $15-40 \%$ of fruit and flower postharvest deterioration ${ }^{2}$. Traditionally, this pathogen is controlled using fungicidal treatments. However, the risk of fungi developing fungicidal resistance limits the use of such treatments ${ }^{3}$. In addition, it is undesirable for fresh fruits and crops to contain fungicidal residues ${ }^{4}$. Thus, it is more feasible to increase plant stress resistance at the molecular level.

\footnotetext{
Correspondence: Ningjia He (hejia@swu.edu.cn)

'State Key Laboratory of Silkworm Genome Biology, Southwest University, Beibei, Chongqing 400715, China

${ }^{2}$ College of Forestry, Shandong Agricultural University, Taian, Shandong 271018, China
}

Methylation plays an important role in plant stress resistance. For instance, in Arabidopsis, DNA methylation controls defense responses to Pseudomonas syringae, and the overall destruction of methyl groups on DNA activates these responses ${ }^{5}$. The resistance of Arabidopsis $d c l$ or $r d r$ mutants to beet cyst nematodes (Heterodera schachtii) is stronger than that of the wild type ${ }^{6}$. Tobacco mosaic virus-induced DNA hypomethylation of LRRs is related to increased genomic rearrangements at these sites $^{7}$. In Medicago truncatula, the differences in the transcript levels of the resistance gene REP1 are related to the methylation state of its promoter region, and REP1 is associated with resistance to Erysiphe pisi ${ }^{8}$.

DNA methylation is a conserved form of epigenetic marking that is related to immunity, genome stability, imprinting, and environmental responses ${ }^{9,10}$. In plants, DNA methylation occurs in two contexts, symmetric (CG and $\mathrm{CHG}$ ) and asymmetric $(\mathrm{CHH})$, in which $\mathrm{H}$ may be A, $\mathrm{T}$, or C. In Arabidopsis, METHYLTRANSFERASE1 (MET1) maintains CG methylation (mCG), CHROMOMETHYLASE (CMT) 3 maintains CHG methylation

\section{(c) The Author(s) 2021}

(c) (i) Open Access This article is licensed under a Creative Commons Attribution 4.0 International License, which permits use, sharing, adaptation, distribution and reproduction cc) in any medium or format, as long as you give appropriate credit to the original author(s) and the source, provide a link to the Creative Commons license, and indicate if changes were made. The images or other third party material in this article are included in the article's Creative Commons license, unless indicated otherwise in a credit line to the material. If material is not included in the article's Creative Commons license and your intended use is not permitted by statutory regulation or exceeds the permitted use, you will need to obtain permission directly from the copyright holder. To view a copy of this license, visit http://creativecommons.org/licenses/by/4.0/. 
(mCHG), and CMT2 maintains $\mathrm{CHH}$ methylation $(\mathrm{mCHH})^{10,11}$. DOMAINS REARRANGED METHYLTRANSFERASE (DRM) 2 establishes de novo DNA methylation in three contexts through the RNA-directed DNA methylation (RdDM) pathway. The RdDM pathway has two main steps: siRNA biogenesis and siRNA-guided DNA methylation. The first step involves RNA polymerase (Pol) IV and DICER-LIKEs (DCLs), whereas the second step involves Pol V, ARGONAUTE4/6 (AGO4/6), and DRMs ${ }^{12,13}$. To balance genome methylation and maintain gene expression, plants utilize DNA demethylase to eliminate methylcytosines and replace them with unmethylated cytosine. Demeter (DME) and REPRESSOR OF SILENCING1 (ROS1) are involved in this process ${ }^{9,14}$.

Transposable elements (TEs) are the main sources of small RNAs in plants. Therefore, the insertion of TEs in genes is easily regulated by the RdDM pathway ${ }^{15}$. The activation of TEs can regulate gene expression, and the activity of TEs is regulated by DNA methylation ${ }^{16}$. In rice PigmS promoters, the methylation levels of TEs regulate expression in a tissue-specific manner and balance a highresistance phenotype with low yield loss ${ }^{17}$. The methylation states of TEs may be key factors in regulating multiple genes simultaneously. Therefore, regulating the methylation of TEs may be an effective breeding strategy to improve desirable agronomic traits and diminish undesirable agronomic traits ${ }^{18}$.

There have been limited studies on the role of DNA methylation in plant-pathogen interactions, especially those in woody plants. Mulberry (Morus L.) is a cultivated fruit crop of the Moraceae family. Mulberry fruit is popular in Asia because of its good taste and high nutritional value. Mulberry is also rich in pharmacological components, including active compounds such as flavonoids and polysaccharides that have anti-inflammatory and hypoglycemic effects ${ }^{19}$. However, mulberry plants are often infected with a variety of diseases; $B$. cinerea is a main pathogen of mulberry ${ }^{20}$. The genome of Morus notabilis C.K. Schneid is relatively small (approximately $330 \mathrm{Mb}$ ) and has been completely sequenced ${ }^{21}$. More than half of the mulberry genome is composed of TEs, including Copia (10.44\%), Gypsy (9.20\%), Lard (8.59\%), Trim (0.61\%), L1 (0.12\%), RTE (0.29\%), PIF-Harbinger (1.90\%), CMC (2.37\%), hAT (2.88\%), MuLE (0.38\%), MITE (13.83\%), and Helitron $(0.98 \%)^{22,23}$.

In this study, single-base resolution DNA methylation was generated from the leaves of $M$. notabilis plants mock-treated or inoculated with $B$. cinerea. DNA methylation in mulberry leaves changed dynamically after infection with $B$. cinerea. Compared with the mock samples, the inoculated samples showed decreased mCG and $\mathrm{mCHG}$ levels and increased $\mathrm{mCHH}$ levels. Many resistance-related genes showed reduced methylation, whereas metabolism-related genes showed increased methylation. The application of DNA methylation inhibitors resulted in the hypomethylation of resistancerelated genes and increased resistance to $B$. cinerea, indicating that the reduced level of DNA methylation is highly significant with regard to resistance to $B$. cinerea. The expression levels of resistance genes in mulberry were correlated with the expression of MET1, which encodes a product that maintains mCG. The silencing of MnMET1 induced by tobacco curly shoot virus (TbCSV) resulted in increased resistance of mulberry to $B$. cinerea. Notably, the promoter regions of many resistance genes were hypomethylated, which may explain the epigenetic regulation of resistance to $B$. cinerea in mulberry. In addition, although the MITEs in mulberry account for only $13.2 \%$ of the genome, they are the most important TE-regulating genes expressed through the RdDM pathway, which is the source of 24-nt siRNAs. In summary, our study revealed for the first time the DNA methylation dynamics of a woody plant genome in response to $B$. cinerea.

\section{Results \\ DNA methylome of mulberry leaves}

To analyze mulberry methylomes, we conducted wholegenome bisulfite sequencing and generated single-base DNA methylation maps for mulberry leaves. For the mock treatment (mock) and the $B$. cinerea inoculation treatment (inoculated), DNA from three biological leaf replicates was sequenced (Fig. 1a). The genome of M. notabilis is approximately $330 \mathrm{Mb}(2 n=14)$. Each sequencing library produced approximately $100 \mathrm{M}$ paired-end reads (100-500 bp), covering approximately $80 \%$ of the genome. For each library, the conversion rate was approximately 99.5\% (Table S1). The sequencing depth reached 50×, indicating that our sequencing data were of sufficient quality for further analyses.

Averaged across the genome, the percentages of mCG, $\mathrm{mCHG}$, and $\mathrm{mCHH}$ in the total $\mathrm{mC}$ sites were $35.5,26.6$, and $37.9 \%$, respectively, in the mock samples and 33.8 , 25.5 , and $40.7 \%$, respectively, in the inoculated samples (Fig. 1b). Compared with the mock samples, the inoculated samples showed significantly decreased mCG, slightly decreased mCHG, and significantly increased $\mathrm{mCHH}$ levels. Thus, dynamic methylation changes occurred in mulberry in response to $B$. cinerea infection.

Next, we analyzed the average DNA methylation levels of genes and TEs (Fig. 1c, d). In genes, the mCG level was higher in both the gene bodies and their flanking regions, similar to previous findings in soybean ${ }^{24}$ and sugar beet ${ }^{25}$, while the mCHG and $\mathrm{mCHH}$ levels were lower in the gene bodies but higher in the flanking regions. Adjacent to the genes, the levels of $\mathrm{mCG}$, $\mathrm{mCHG}$, and $\mathrm{mCHH}$ were low. However, as the distance from the genes increased, so 

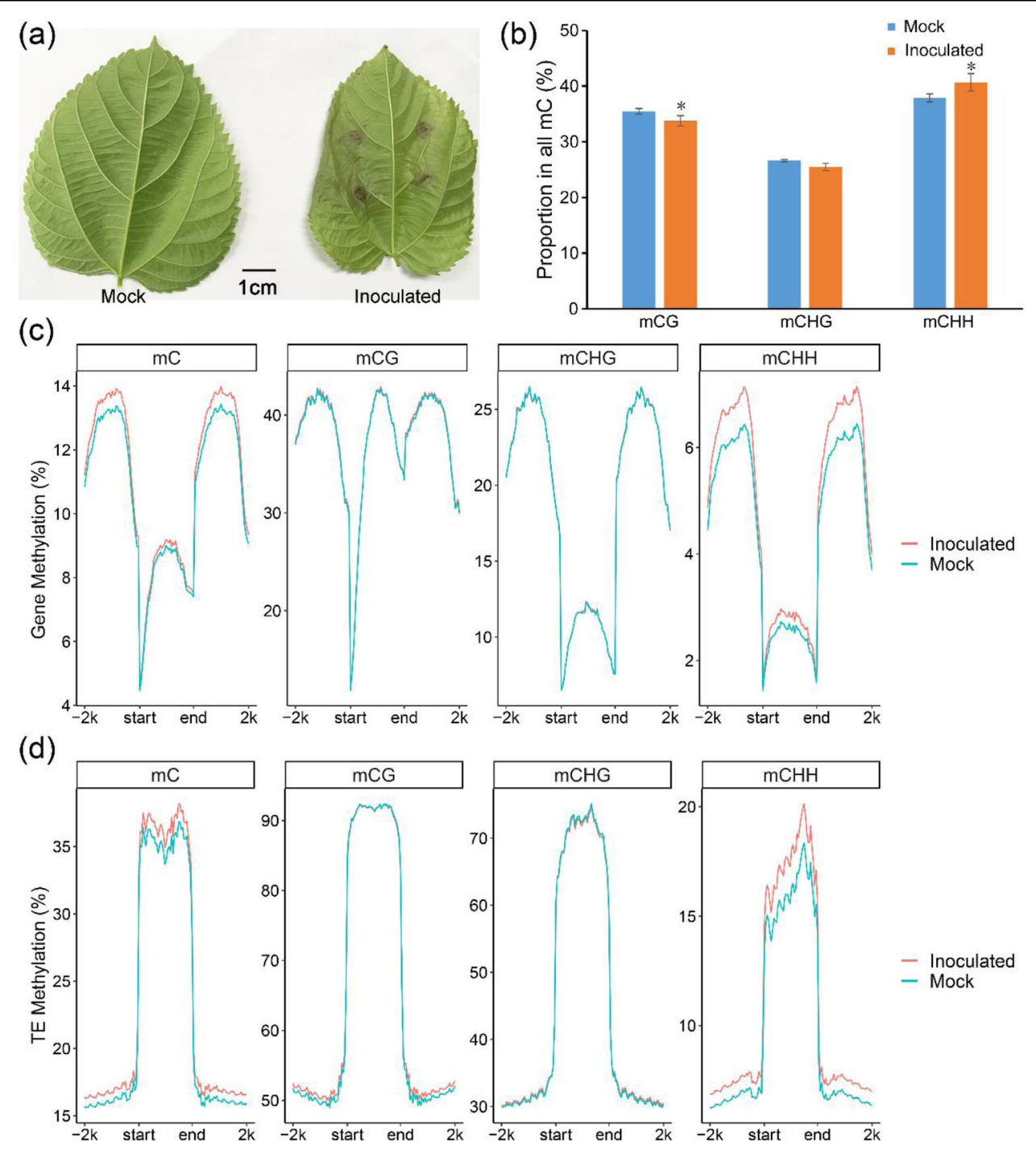

Fig. 1 Characterization of mulberry methylomes. a Photographs of mock-treated (Mock) and B. cinerea-inoculated (Inoculated) mulberry leaves. b Proportions of $\mathrm{mCG}, \mathrm{mCHG}$, and $\mathrm{mCHH}$ in all the methylcytosine in Mock and Inoculated leaves; error bars indicate SDs, $n=3$ ( ${ }^{*}$-value $<0.05$, twotailed t-test). Levels of $\mathrm{mC}, \mathrm{mCG}, \mathrm{mCHG}$, and $\mathrm{mCHH}$ surrounding the upstream, gene body, and downstream regions of the genes (c) and TEs (d) in two samples. The mean values of three biological replicates are shown

did the levels of mCG, mCHG, and $\mathrm{mCHH}$. In the TEs, the levels of $\mathrm{mCG}, \mathrm{mCHG}$, and $\mathrm{mCHH}$ were much higher in the gene bodies than in the flanking regions, similar to previous findings in orange ${ }^{26}$ and strawberry ${ }^{27}$. Because TEs play important roles in genome evolution and gene regulation, we analyzed the individual methylation patterns of different TE superfamilies, including retroTEs (Copia, Gypsy, Lard, Trim, L1, and RTE) (Fig. S1) and DNA TEs (PIF-Harbinger, CMC, hAT, MuLE, MITE, and Helitron) (Fig. S2). The methylation model of MITE was consistent with that of a TE, indicating that MITE plays a leading role in TE methylation (Fig. 1d and Fig. S2). Interestingly, compared with other TEs, MITE showed significantly higher $\mathrm{mCHH}$ levels, which is indicative of the potential role of asymmetric methylation in MITE silencing.

\section{Expression profiles of genes related to DNA methylation and the RdDM pathway}

Genomic DNA methylation can be maintained through the interaction of DNA methylation with DNA demethylation processes. To analyze the mechanisms of dynamic DNA methylation in mulberry resistance to $B$. cinerea, we first detected the expression levels of DNA demethylase genes, including $M n D M E$ and $M n D M L$. Their transcript levels were not significantly different between the mock and inoculated samples (Fig. S3). In Arabidopsis, mCG, mCHG, and $\mathrm{mCHH}$ are maintained 

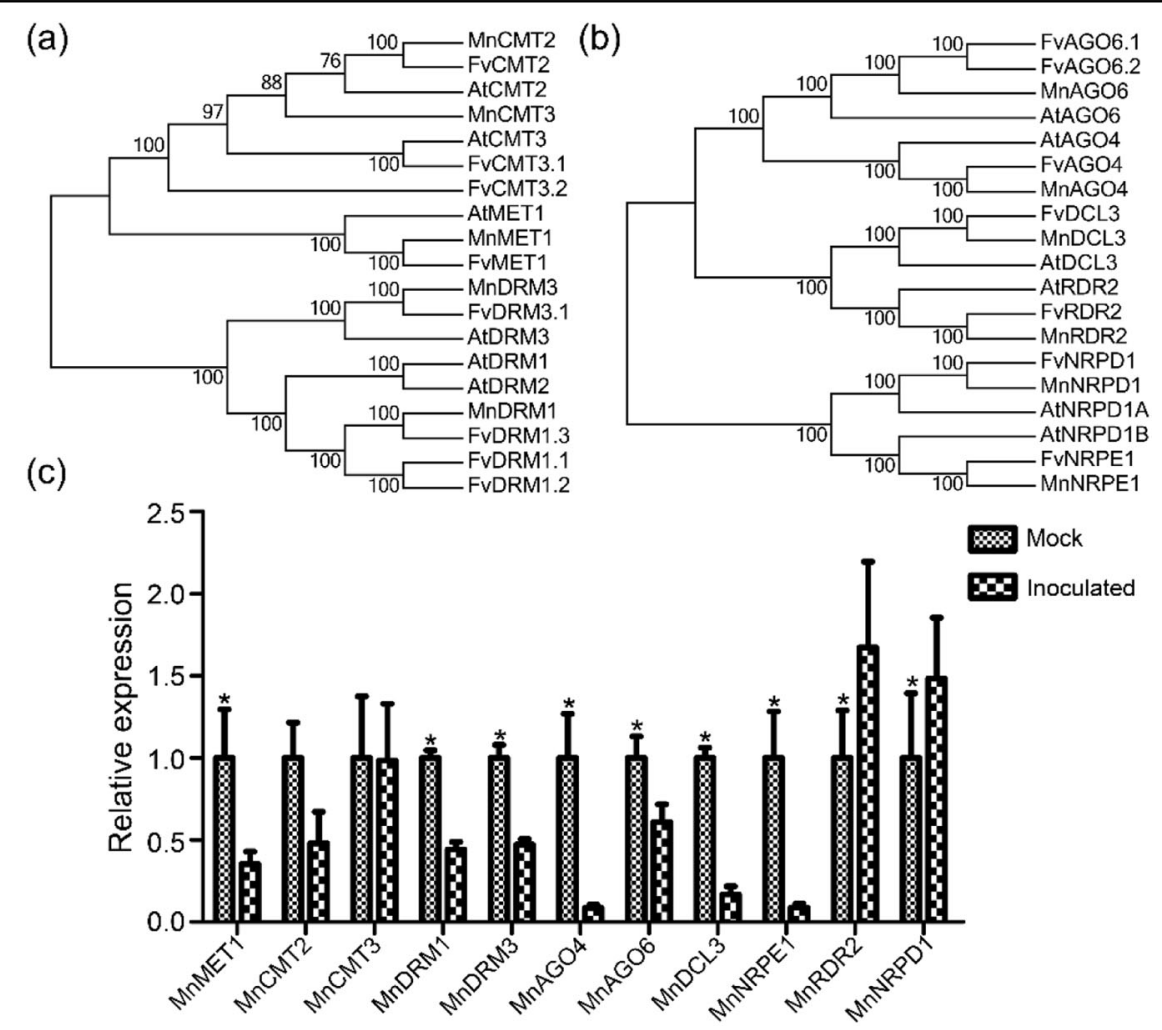

Fig. 2 Expression of genes involved in DNA methylation. Phylogenetic analyses of DNA methyltransferase (a) and RdDM (b) genes in mulberry, strawberry, and Arabidopsis. The accession numbers of the CDSs are as follows: AtDRM1 (AT5G15380), AtDRM2 (AT5G14620), AtDRM3 (AT3G17310), AtMET1 (AT5G49160), AtCMT2 (AT4G19020), AtCMT3 (AT1G69770), FvDRM1.1 (gene05866), FvDRM1.2 (gene06047), FvDRM1.3 (gene28439), FvDRM3.1 (gene17910), FvMET1 (gene13037), FvCMT2 (gene13664), FvCMT3.1 (gene10077), FvCMT3.2 (gene15171), MnCMT2 (KE346101.1), MnCMT3 (KE343837.1), MnMET1 (KE344409.1), MnDRM3 (KE344683.1), MnDRM1 (KE345913.1), AtAGO4 (AT2G27040), AtAGO6 (AT2G32940), AtDCL3 (AT3G43920), AtNRPD1A (AT1G63020), AtNRPD1B (AT2G40030), AtRDR2 (AT4G11130), FvAGO4 (gene07657), FvAGO6.1 (gene16926), FvAGO6.2 (gene16928), FvDCL3 (gene15481), FvNRPD1 (gene32373), FvNRPE1 (gene14287), FvRDR2 (gene32159), MnAGO4 (KE344194.1), MnAGO4 (KE346072.1), MnDCL3 (KE344662.1), MnNRPD1 (KE345823.1), MnNRPE1 (KE344454.1), and MnRDR2 (KE345786.1). c qRT-PCR analyses of DNA methylation genes. All the expression levels were normalized to the expression of the mulberry actin gene. Error bars indicate $\mathrm{SDs}, n=3\left({ }^{*} P\right.$-value $<$ 0.05 , two-tailed t-test)

by MET1, CMT3, and CMT2, respectively, and all three can be de novo methylated by DRMs through the RdDM pathway ${ }^{9,10}$. To further understand why DNA methylation patterns change, we compared the transcript levels of genes involved in DNA methylation and the RdDM pathway between the mock and inoculated samples. Five DNA methyltransferase genes were identified in the mulberry genome: $M n C M T 2, M n C M T 3, M n M E T 1$, MnDRM1, and MnDRM3 (Fig. 2a). Compared with those in the mock samples, the transcript levels of all these genes, except $M n C M T 3$, in the inoculated samples were lower (Fig. 2c). The downregulation of MnDRM1 and $M n D R M 3$ suggested that $\mathrm{RdDM}$ activity might decrease during $B$. cinerea infection. To test this hypothesis, we detected the transcript levels of RdDMrelated genes. RdDM consists of two stages: siRNA production, which requires Pol IV, RDR2, and DCL3, and siRNA-mediated DNA methylation, which requires
Pol V, AGO4/6, and DRMs. We found genes encoding the largest subunits of Pol IV (MnNRPD1) and Pol V (MnNRPE1), as well as MnRDR2, MnDCL3, MnAGO4, and $M n A G O 6$, in the mulberry genome (Fig. 2b). Compared with those in the mock samples, the transcript levels of MnRDR2 and MnNRPD1 in the inoculated samples significantly increased, whereas the transcript levels of the other genes significantly decreased (Fig. 2c).

\section{Analyses of siRNA expression and DNA methylation}

The RdDM pathway is usually dependent on 24-nt siRNAs. Therefore, we studied the expression profiles of small RNAs in the mock and inoculated samples using high-throughput sequencing. Among the small RNAs, lengths of 21-nt and 24-nt were the most abundant in the mulberry samples (Fig. 3a). In the mock samples, the latter was the most abundant type of small RNA, whereas, 


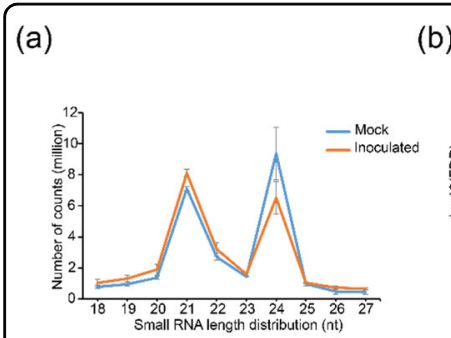

(c)

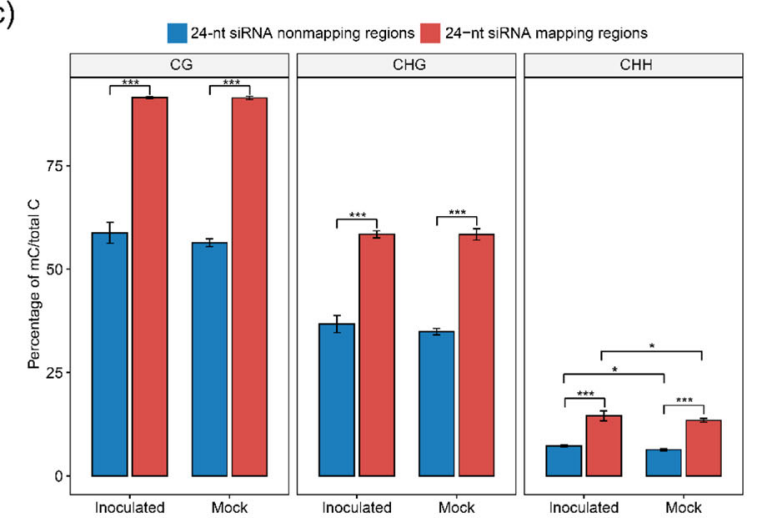

(d)

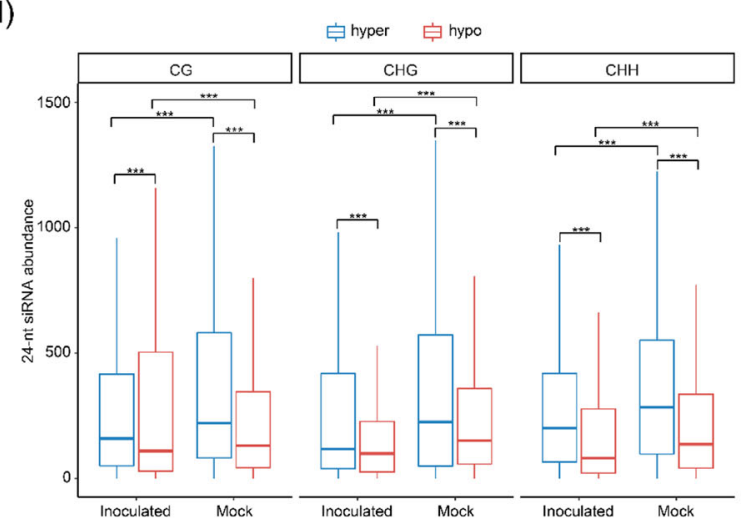

Fig. 3 DNA methylation and 24-nucleotide siRNAs. a Size

distribution of the sequenced small RNAs in mulberry. Three biological replicates are shown. $\mathbf{b}$ Volcano plot indicating the up- and downregulated 24-nt siRNAs between the two groups. c DNA methylation levels in mapped regions with 24-nt siRNAs compared with those in mapped regions without 24-nt siRNAs in mock-treated (Mock) and B. cinerea-inoculated (Inoculated) mulberry leaves. Error bars indicate SDs, $n=3\left({ }^{*} P\right.$-value $<0.05$, ${ }^{* *} P$-value $<0.001$, two-way ANOVA). $\mathbf{d}$ Abundance of 24-nt siRNAs located in the $\mathrm{CG}, \mathrm{CHG}$, and $\mathrm{CHH}$ hypermethylated and hypomethylated regions ( ${ }^{* *} P$-value $<$ 0.001, two-way ANOVA)

in the inoculated samples, the former was the most abundant. Compared with the mock samples, the inoculated samples had significantly lower amounts of 24-nt small RNAs. Next, the expression levels of 24-nt siRNAs were compared between the mock and inoculated samples (Fig. 3b). In total, 1,982 differentially expressed 24-nt siRNAs (adjusted $P$-value $<0.01$ ), including 320 upregulated 24-nt siRNAs and 1,662 downregulated 24-nt
siRNAs, were identified between the inoculated and mock samples.

To further explore the correlations between 24-nt siRNAs and methylation, we compared the average methylation levels between regions with and without mapped 24-nt siRNAs in the mock and inoculated samples (Fig. 3c). The levels of mCG, mCHG, and $\mathrm{mCHH}$ were significantly greater in the regions with mapped siRNAs than in the regions without mapped siRNAs. Compared with those in the mock samples, the $\mathrm{mCHH}$ levels in the inoculated samples were significantly greater. Interestingly, in the mock samples, more 24-nt siRNAs were present in CG-, CHG-, and CHH-hypermethylated regions than in hypomethylated regions (Fig. 3d), whereas fewer 24-nt siRNAs were present in CGhypermethylated regions than in hypomethylated regions in the inoculated samples. Compared with those in the mock samples, the abundance levels of 24-nt siRNAs were significantly reduced in all the inoculated samples, except for the CG hypomethylation regions. We analyzed the distributions of 24-nt siRNAs on gene bodies, TE bodies, and their flanking regions (Fig. 4a, b). The 24-nt siRNAs of mulberry were preferentially located on the flanking regions of genes but were more abundant on $\mathrm{TE}$ bodies than on their flanking regions. Interestingly, 24-nt siRNAs were concentrated mainly at the ends of TE bodies. Compared with the mock samples, the inoculated samples had fewer 24-nt siRNAs on gene promoters, $3^{\prime}$ regulatory regions, and TE bodies. We further analyzed the distribution of 24-nt siRNAs in each TE superfamily (Figs. S4 and S5). The 24-nt siRNA distribution on TEs was concentrated mainly in the MITE superfamily, indicating that MITE plays an important role in generating 24-nt siRNAs.

\section{Differentially methylated regions and responses to $B$. cinerea stress}

To explore the potential functions of methylation in the responses to $B$. cinerea, we analyzed differentially methylated regions (DMRs) in the genome between mock and inoculated mulberry plants (Fig. 5). Compared with mock samples, inoculated samples had 104,957 DMRs, of which 4,288 were mCG, 5,412 were mCHG, and 95,257 were $\mathrm{mCHH}$ (Fig. 5a-c). Among all the DMRs, $77.12 \%$ were hypo-DMRs of mCG, 70.12\% were hypo-DMRs of $\mathrm{mCHG}$, and $21.25 \%$ were hypo-DMRs of $\mathrm{mCHH}$. Most of the CG and CHG DMRs were hypo-DMRs, and most of the CHH DMRs were hyper-DMRs, consistent with previous results (Fig. 1b). In addition, we identified some of the DMRs (Fig. S6).

Methylation in promoter regions regulates gene expression, and promoter methylation is negatively correlated with gene expression. To analyze the changes in promoter methylation in mulberry under B. cinerea stress, 

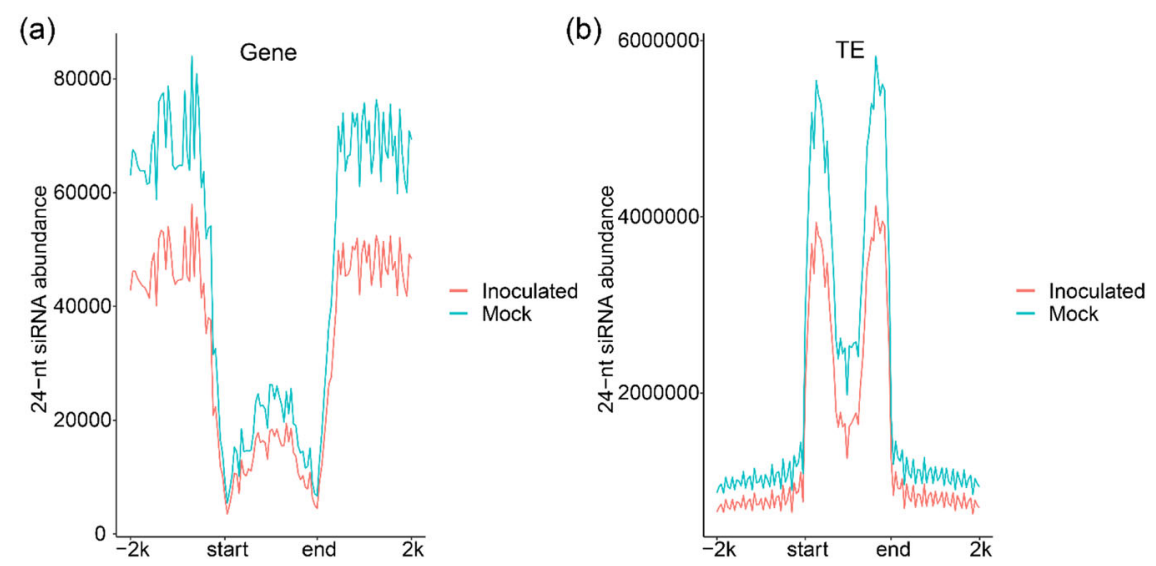

Fig. 4 Distribution of 24-nt siRNAs. The 24-nt siRNA distributions on the gene bodies and flanking regions (a) and TEs (b) of mock-treated (Mock) and B. cinerea-inoculated (Inoculated) mulberry leaves. The mean values of three biological replicates are shown

we searched for differentially methylated promoters (DMPs) situated in the DMRs. The search revealed 976 (811 hypo-DMPs), 1,003 (774 hypo-DMPs), and 12,779 (2,842 hypo-DMPs) DMPs of mCG, $\mathrm{mCHG}$, and $\mathrm{mCHH}$, respectively. To understand the functions of DMPs, Kyoto Encyclopedia of Genes and Genomes (KEGG) pathway enrichment analysis was performed to identify the pathways enriched for genes with DMPs (Fig. $5 d-f$ ). The KEGG pathways showed that plant-pathogen interaction (87.50\% hypo-DMPs) was the main category of genes with CHG-DMPs, carbon metabolism (73.68\% hypo-DMPs) was the main category of genes with CHG-DMPs, and metabolic pathways (21.83\% hypo-DMPs) were the main category of genes with $\mathrm{CHH}-\mathrm{DMPs}$ (Fig. S7a). These results indicated that $\mathrm{mCG}$ and $\mathrm{mCHG}$ may participate in responses to $B$. cinerea by enhancing the expression of genes related to disease resistance and carbon metabolism and that $\mathrm{mCHH}$ may be involved in these responses by inhibiting the expression of genes related to metabolic pathways.

Compared with the mock samples, the inoculated samples had 6,273 differentially methylated genes (DMGs) consisting of 954 (599 hypo-DMGs), 625 (430 hypo-DMGs), and 4,694 (1,117 hypo-DMGs) DMGs of mCG, mCHG, and $\mathrm{mCHH}$, respectively, located in DMRs (Fig. S8). Interestingly, the KEGG pathway analysis showed that metabolic pathways contained the most genes with differential CG, CHG, and CHH methylation levels between the mock and inoculated samples. In the metabolic pathway category, hypo-DMGs accounted for $52.90,71.72$, and $26.84 \%$ of the total DMGs of mCG, $\mathrm{mCHG}$, and $\mathrm{mCHH}$, respectively (Fig. S7b). This suggests that the dynamic differential methylation of genes related to metabolic pathways may play a key role in the response to $B$. cinerea. We performed a KEGG enrichment analysis of the differentially expressed genes (DEGs) between inoculated and mock samples identified from the transcriptome data (Fig. S9) and found that the DEGs were associated mainly with plant-pathogen interaction, MAPK signaling, and plant hormone signal transduction. These findings suggest that all of these pathways are involved in responses to $B$. cinerea stress in mulberry.

\section{Gene and TE DMRs}

We analyzed the proportions of CG, CHG, and $\mathrm{CHH}$ DMRs that overlapped with genes and TEs (Fig. 6a). The proportions of DMRs, especially $\mathrm{CHG}$ and $\mathrm{CHH}$, had significantly greater overlaps with TEs than with genes, and $63.59 \%$ of the CHH hyper-DMRs overlapped TEs. Further analyses showed that the DMRs overlapped mainly with the MITE superfamily of TEs (Fig. 6b). Gene promoter regions affect transcriptional regulation, and differences in promoter methylation affect transcriptional expression. The proportions of $\mathrm{CG}, \mathrm{CHG}$, and $\mathrm{CHH}$ DMPs that overlapped TEs (Fig. 6c) were 40.60, 43.13, and $67.22 \%$, respectively. Next, we analyzed the distributions of DMPs that overlapped with each TE superfamily (Fig. S10). DMPs overlapped mainly with the MITE superfamily. Furthermore, we calculated the proportions of methylation changes in gene and promoter regions that were negatively correlated with changes in gene transcription levels (the proportions of transcripts that increased with decreased methylation and decreased with increased methylation) (Fig. 6d). Approximately 50\% of methylation changes were negatively correlated with changes in gene transcription levels. This indicated that the methylation of both the gene body and the promoter region affects gene expression.

\section{Effects of DNA methylation on gene expression}

To determine how DNA methylation affects mulberry resistance, we applied the DNA methylation inhibitor 5- 


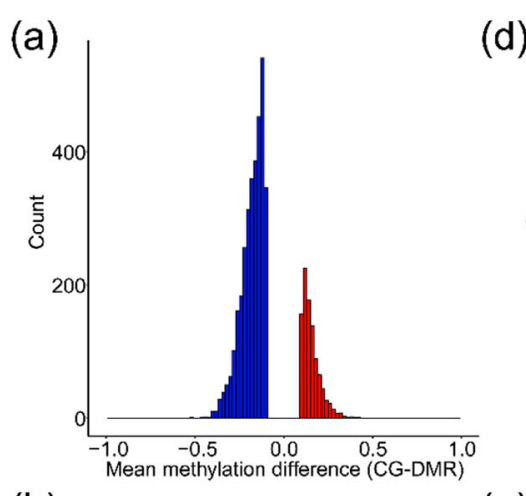

(b)

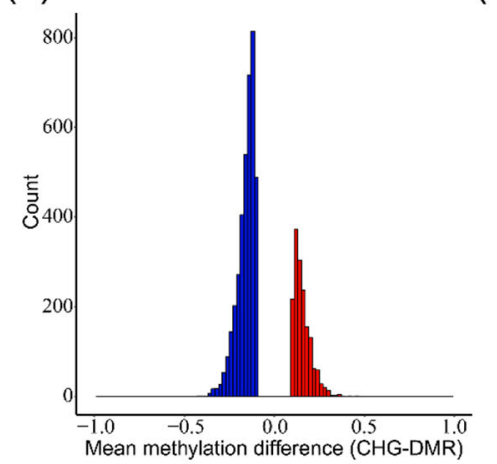

(c)

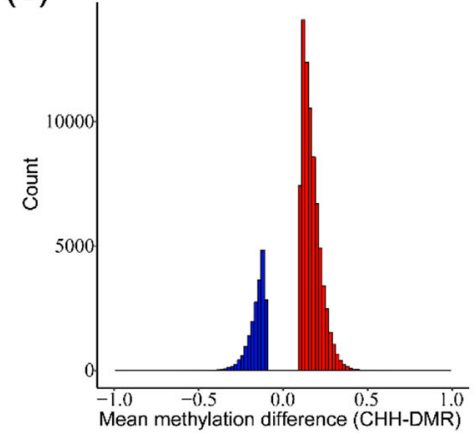

(f) (d)

(e)
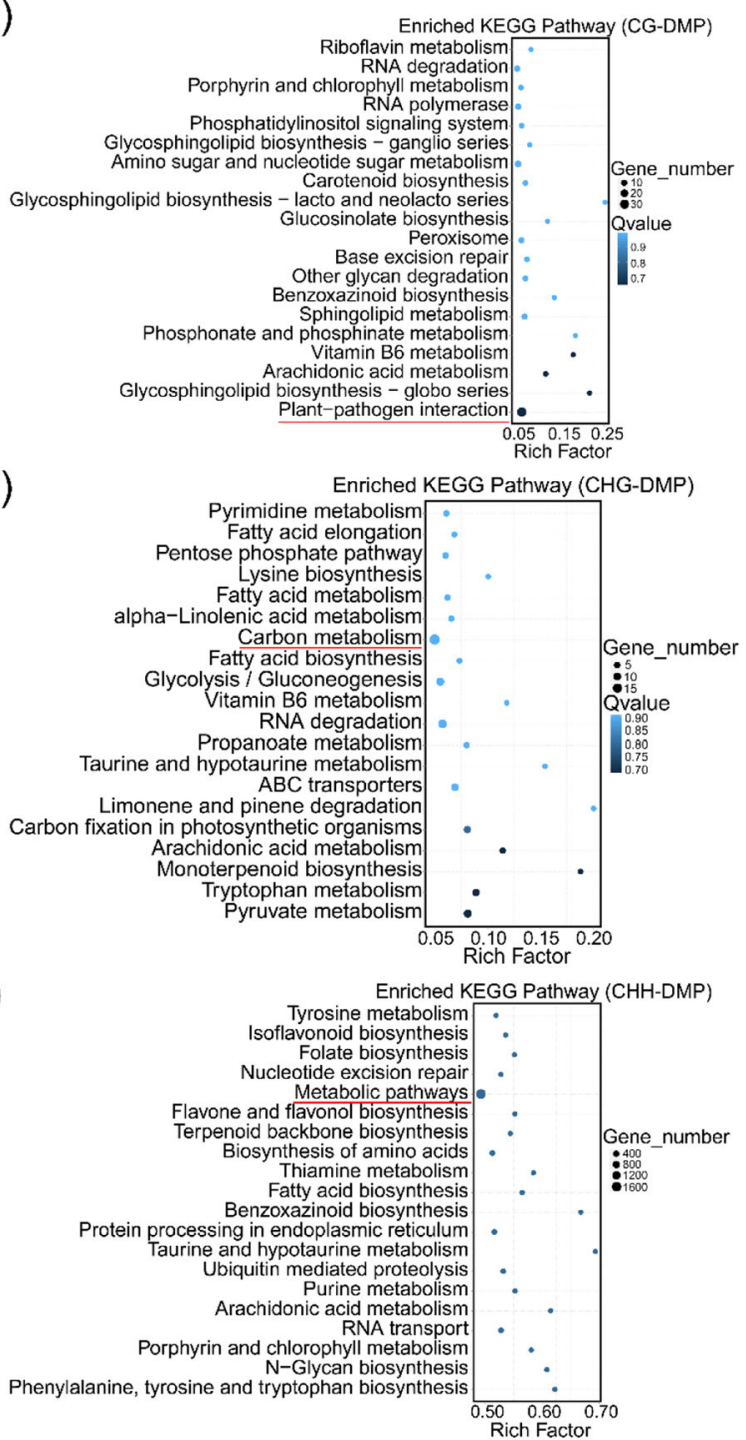

Fig. 5 DMR and KEGG pathway enrichment analyses of DMPs between mock-treated (Mock) and B. cinerea-inoculated (Inoculated) mulberry leaves. Hypo- and hyperdifferentially methylated region counts of CG (a), CHG (b), and CHH (c). Enriched KEGG pathways of the differentially methylated promoters in $\mathrm{CG}(\mathbf{d}), \mathrm{CHG}(\mathbf{e})$, and $\mathrm{CHH}(\mathbf{f})$. The most enriched pathways are underlined in red

azacytidine to mulberry leaves. To confirm that the inhibitor treatment changed the DNA methylation of resistance genes in mulberry, we used methylationsensitive McrBC-PCR to detect the DNA methylation levels of two resistance-related genes, Morus017734 (DMG), encoding the CYSTM domain-containing protein, and Morus025913 (DMP), encoding an LRR receptor-like serine/threonine-protein kinase (Fig. 7a, b). The enzyme McrBC, which cuts methylated DNA but not unmethylated DNA, was used to cut the genomic DNA. Then, McrBC-PCR was conducted using the digested DNA as the template. The methylation-sensitive McrBCPCR analyses showed that both genomic regions were highly methylated, consistent with the whole-genome bisulfite sequencing results. The DNA methylation levels were decreased significantly in the 5-azacytidine-treated samples (Fig. 7c, d). To verify the effects of decreased methylation levels on gene expression, we determined the expression levels of these two genes. Compared with the mock samples, the inoculated samples showed significantly decreased methylation levels in the two genomic regions and the significant upregulation of these two genes (Fig. 7e). In the 5-azacytidine-treated samples, the two genes were also significantly upregulated (Fig. 7f). Next, to explore whether inhibiting methylation enhanced mulberry resistance to $B$. cinerea, mulberry leaves treated with or without 5-azacytidine were inoculated with $B$. cinerea (Fig. S11). The untreated leaves developed severe 


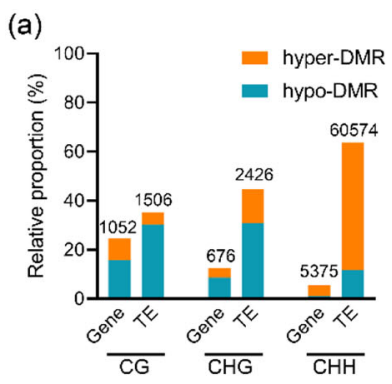

(c)

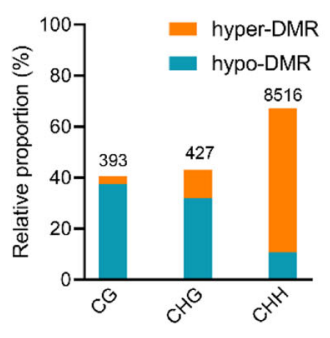

(b)

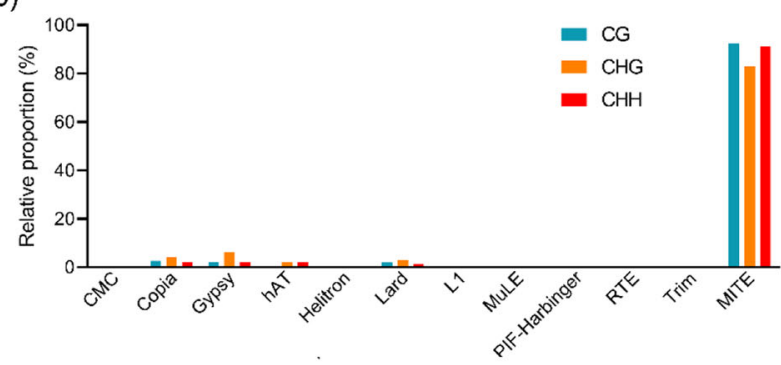

(d)

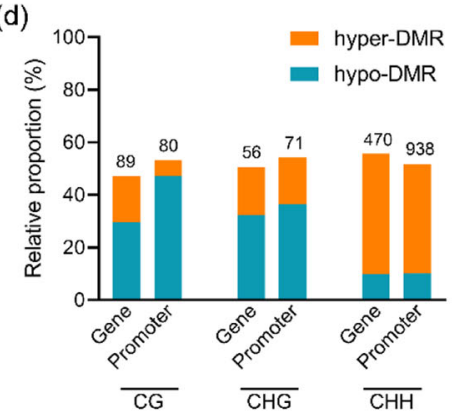

Fig. 6 Differentially methylated regions of genes and TEs. a The relative proportions of hypo- and hyperdifferentially methylated regions that overlapped with genes and TEs. $\mathbf{b}$ The relative proportions of differentially methylated regions in CG, CHG, and CHH that overlapped with TEs in each superfamily. $\mathbf{c}$ The relative proportions of differentially methylated promoters in CG, CHG, and CHH that overlapped with TEs. $\mathbf{d}$ The proportions of transcripts that increased with decreased methylation and decreased with increased methylation. The number at the top of the figure represents the absolute number of DMRs

necrotic lesions by 3 days postinoculation, while those treated with 5-azacytidine developed mild necrotic lesions (Fig. S11a). A quantitative analysis showed that the growth of B. cinerea was inhibited in the 5-azacytidinetreated leaves but not in the untreated leaves (Fig. S11b).

\section{MnMET1 silencing in mulberry enhanced resistance to $B$. cinerea}

Because the 24-nt siRNA abundance and mCG levels in the promoter regions of resistance-related genes were reduced in the inoculated samples, we conducted virusinduced gene silencing (VIGS) of MnAGO4 and MnMET1 independently in mulberry. Phytoene desaturase (PDS) was used as a positive control (Fig. S12). The colors of plants injected with $\mathrm{MMDaV}+2 \mathrm{mDNA} 1-\mathrm{MnPDS}$ (at 14 days after VIGS treatment) were significantly paler than those of plants injected with the control construct, MMDaV+2mDNA1 (Fig. S12a). Compared with the control plants (injected with $M M D a V+2 m D N A 1$ ), the plants treated with $M M D a V+2 m D N A 1-M n P D S$ showed silencing of PDS expression in their second leaves, as confirmed by gene-specific qRT-PCR analysis. The significantly decreased transcript level of MnPDS confirmed that its expression was effectively inhibited by VIGS (Fig. S12b). Next, the transcript levels of MnAGO4 and $M n M E T 1$ were detected in the MMDaV+2mDNA1 control group, as well as in the $M M D a V+2 m D N A 1-$ $M n A G O 4$ and MMDaV+2MDNA1-MnMET1 treatment groups. We detected significant reductions in the transcript levels of MnAGO4 in the MMDaV+2mDNA1$M n A G O 4$ treatment group and of MnMET1 in the $M M D a V+2 M D N A 1-M n M E T 1$ treatment group (Fig. 8a, b). To evaluate the resistance of mulberry to $B$. cinerea after the silencing of $M n A G O 4$ and $M n M E T 1$, the leaves of gene-silenced and control mulberry lines were inoculated with $B$. cinerea. The control leaves developed severe dry necrosis by 3 days post inoculation, whereas the leaves of $M n A G O 4$-silenced plants showed slightly milder symptoms and those of $M n M E T 1$-silenced plants developed only mild necrotic lesions (Fig. 8c). A quantitative analysis showed that compared with the control plants, those with silenced $M n A G O 4$ expression did not show significantly inhibited $B$. cinerea growth, but those with silenced MnMET1 expression showed significantly inhibited B. cinerea growth (Fig. 8d). In addition, Morus002632, which encodes the resistance-related LRR receptor-like serine/threonine-protein kinase, was expressed in $M n M E T 1$-silenced plants but not in the control or MnAGO4-silenced plants (Fig. S12c). Further analyses of methylation in the promoter region of Morus002632 showed that the methylation level in the $M n M E T 1$-silenced plants was decreased compared with that in the control and MnAGO4-silenced plants (Fig. S12d). This indicated that the silencing of MnMET1 reduced methylation and enhanced the expression of 


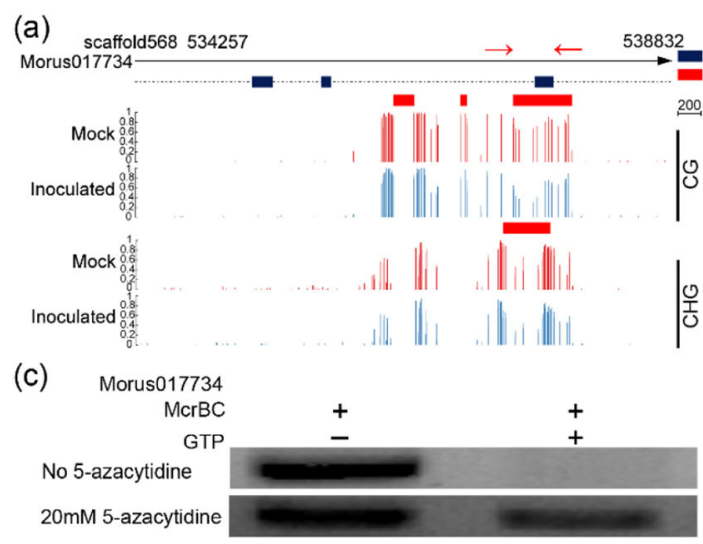

(b)

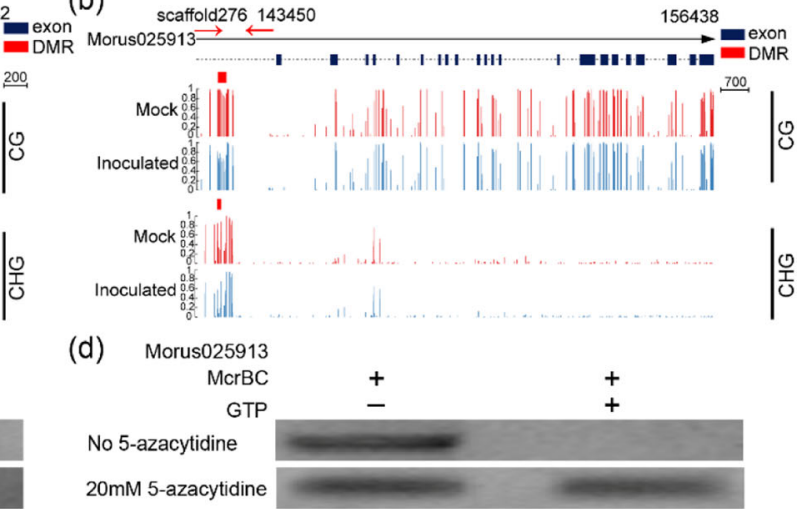

(e)

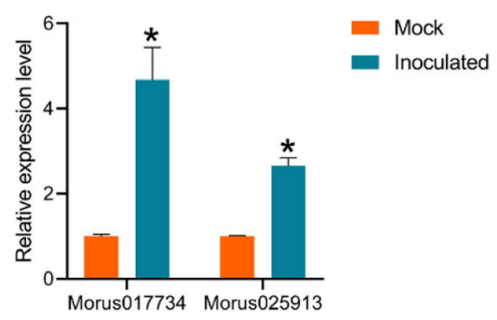

(f)

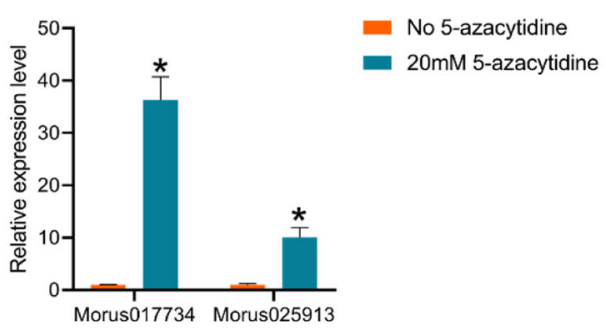

Fig. 7 Effects of DNA methylation on the expression of mulberry-resistance genes. DNA methylation surrounding Morus017734 (a) and Morus025913 (b) in mock-treated (Mock) and B. cinerea-inoculated (Inoculated) mulberry leaves. The red rectangles represent DMRs. The red arrows represent the regions verified by McrBC-PCR. McrBC-PCR analyses of Morus017734 (c) and Morus025913 (d). + and - indicate the presence and absence of GTP, respectively. e The expression of Morus017734 and Morus025913 in mock-treated (Mock) and B. cinerea-inoculated (Inoculated) mulberry leaves. $\mathbf{f}$ The expression of Morus017734 and Morus025913 in mulberry leaves treated with and without 5-azacytidine. All the expression levels were normalized to the expression of the mulberry actin gene. Error bars indicate SDs, $n=3$ ( ${ }^{*} P$-value $<0.05$, two-tailed $t$-test)

resistance genes in mulberry, resulting in enhanced resistance to B. cinerea.

To further verify the role of MnMET1 in mulberry resistance, MnMET1was transiently overexpressed (Fig. S13). We detected a significant increase in the transcript level of MnMET1 in the pLGNL-MnMET1 treatment group (Fig. S13a). To evaluate the resistance of mulberry to $B$. cinerea after transient $M n M E T 1$ overexpression, transiently overexpressed and control mulberry leaves were inoculated with B. cinerea (Fig. S13b). The control leaves showed mild necrotic lesions 2 days after inoculation, whereas the leaves of plants transiently overexpressing MnMET1 showed more necrotic lesions. A quantitative analysis showed that, compared with that of control plants, the resistance of plants transiently overexpressing MnMET1 to B. cinerea was significantly reduced (Fig. S13c).

\section{Discussion}

Genome-wide methylome analyses performed through bisulfite sequencing have been applied to diverse plants, such as Arabidopsis ${ }^{28}$, rice $^{29}$, tomato $^{30}$, apple ${ }^{31}$, poplar ${ }^{32}$, and spruce ${ }^{33}$. Here, cytosine methylation in mulberry was analyzed through genome-wide bisulfite sequencing. We compared the dynamic methylation (hypomethylated and hypermethylated sites) between mock-inoculated and $B$. cinerea-inoculated plants. The proportions of mCG, $\mathrm{mCHG}$, and $\mathrm{mCHH}$ in the total $\mathrm{mC}$ sites were 35.5, 26.6, and $37.9 \%$, respectively, in the mock samples. In Poaceae, $\mathrm{mCHH}$ is usually depleted in deep heterochromatin and abundant in gene-rich regions ${ }^{34}$. In contrast, in mulberry, $\mathrm{mCHH}$ was less abundant in gene-rich regions and more abundant in TE-rich regions (Fig. 1c, d). TEs account for more than half of the mulberry genome ${ }^{22,23}$. Among all the TEs, MITE plays a dominant role in $\mathrm{CHH}$ methylation; this may be related to its high copy number or high activity. In the mulberry genome, MITE is the most abundant TE.

In the endosperm of Arabidopsis and rice, CG demethylation is accompanied by local $\mathrm{CHH}$ hypermethylation $^{35,36}$. In tomato, global DNA hypomethylation during development is accompanied by $\mathrm{CHH}$ hypermethylation of $\mathrm{TEs}^{30}$. In this study on mulberry, compared with mock samples, inoculated samples showed dynamic changes in methylation, with significantly decreased mCG levels, slightly decreased mCHG levels, and significantly increased $\mathrm{mCHH}$ levels (Fig. 1b). The promoter regions of genes related to resistance were generally hypomethylated, whereas those of genes associated with metabolism were generally hypermethylated (Fig. 5). Hypomethylation 

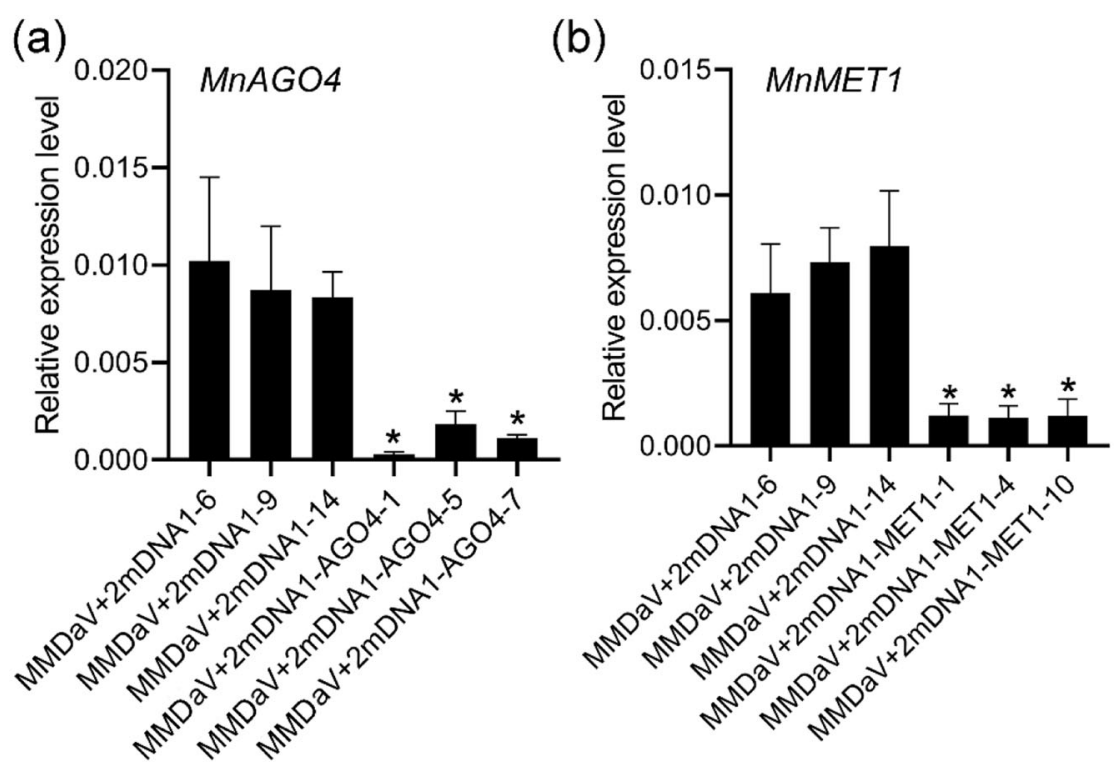

(c)

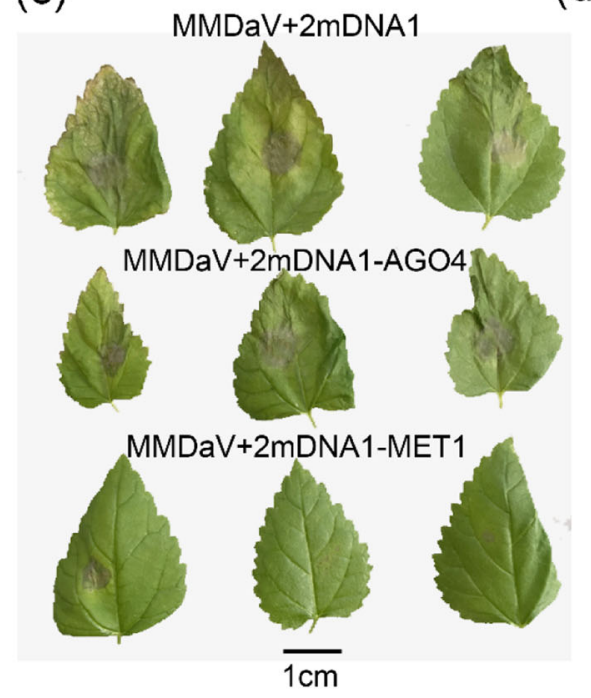

(d)

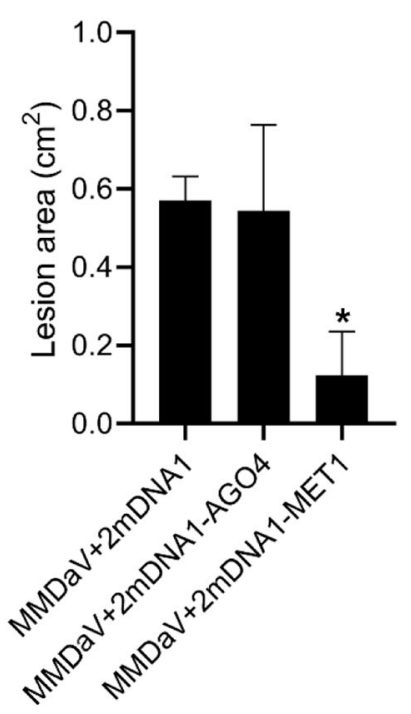

Fig. 8 Silencing of MnMET1 in mulberry leaves resulted in increased resistance. Relative expression levels of MnAGO4 (a) and MnMET1 (b) in mulberry leaves collected from three replicates injected with MMDaV+2mDNA1, MMDaV+2mDNA1-AGO4, and MMDaV+2MDNA1-MET1. All the expression levels were normalized to the expression of the mulberry actin gene. Error bars indicate SDs, $n=3$ ( ${ }^{*} P$-value $<0.05$, two-tailed $t$-test). c Mulberry leaves inoculated with B. cinerea were photographed at 3 days after inoculation. $\mathbf{d}$ Quantitative analyses of the resistance to $B$. cinerea of mulberry leaves injected with MMDaV+2mDNA1, MMDaV+2mDNA1-AGO4, and MMDaV+2MDNA1-MET1

and hypermethylation are beneficial for controlling gene expression in mulberry under B. cinerea stress. Hypermethylation reduces gene transcription, which decreases energy consumption. This means that more energy can be directed to the pathogen defense response. In contrast, the hypomethylation of resistance-related genes increases their expression, resulting in rapid resistance responses to B. cinerea. The activation of TEs is determined mainly by their DNA methylation levels ${ }^{16}$. Genome-wide hypomethylation owing to biological or nonbiological stress activates TEs and increases their fluidity in disease-related genes, thereby regulating gene expression ${ }^{5,37-39}$. In mulberry, the differential methylation of CGs was detected in similar proportions in gene and TE regions, while the differential methylation of $\mathrm{CHGs}$ and $\mathrm{CHHs}$ was detected mainly in TE regions (Fig. 6a). In particular, the differential methylation of $\mathrm{CHHs}$ was detected mainly in $\mathrm{TE}$ regions, indicating that $\mathrm{CHH}$ methylation plays a key role in inhibiting TE activity (Fig. 6b). This also suggests that mulberry TEs may play important roles in regulating gene expression through the RdDM pathway. Transposable elements in promoters regulate gene expression by 
changing their DNA methylation states. Analyses of the methylation states of TEs in mulberry showed that more than $80 \%$ of the differences in methylation occurred in MITEs (Fig. S10). These MITEs tended to be inserted near genes $^{23}$, indicating that MITE may be the most important type of TE regulating gene expression through the RdDM pathway.

DNA methylation is regulated by DNA methylation and demethylation. In Arabidopsis, DNA demethylation is catalyzed by the REPRESSOR OF SILENCING1 family'. These proteins remove $\mathrm{mC}$ bases and cleave the DNA backbone, which is then filled by an unmethylated cytosine. In Arabidopsis, DME contributes to DNA demethylase activity and disease responses ${ }^{40}$, and TE sequences in the promoter regions of resistance-related genes are targets of DNA demethylases ${ }^{41,42}$. Changes in gene promoter methylation in mulberry occurred mainly in TEs (Fig. 6c). Both DNA methylases and demethylases may regulate gene expression mainly by targeting TEs in gene promoter regions. However, DNA demethylase genes were not upregulated in B. cinerea-inoculated mulberry plants (Fig. S3). The role of DNA demethylase genes in mulberry disease resistance remains to be studied further. Instead, we detected the significant downregulation of multiple genes encoding DNA methyltransferases and other key components of the RdDM pathway (Fig. 2). The significant reduction in $M n M E T 1$ transcript levels in $B$. cinerea-inoculated samples compared with mock samples suggested that MET1 is responsible for the significant reduction in mCG. In addition, passive DNA demethylation may occur when the maintenance methyltransferase is inactive during the cell cycle after DNA replication, resulting in the newly synthesized strand remaining in an unmethylated state. It is also possible that DNA demethylation activity is relatively increased owing to the decrease in DNA methyltransferase activity. This relative change may also be responsible for the declines in $\mathrm{mCG}$ and mCHG. In the RdDM pathway, 24-nt siRNAs direct DRM1 and DRM2 to methylate the $\mathrm{CHH}$ sequence context ${ }^{10,43,44}$. The significantly increased transcript levels of MnRDR2 and MnNRPD1 in B. cinerea-inoculated mulberry plants may explain the significant increase in $\mathrm{mCHH}$. However, the abundance of 24-nt siRNAs was significantly decreased in $B$. cinerea-inoculated samples. Thus, the increase in $\mathrm{mCHH}$ in mulberry does not appear to be established and maintained only by the RdDM pathway. The downregulation of $M n D C L 3$ may be a main reason for the decrease in 24-nt siRNA levels. The abundance of 24-nt siRNAs in mulberry was closely related to $\mathrm{CHG}$ and $\mathrm{CHH}$ hypermethylation but less closely related to CG hypermethylation (Fig. 3d). There was no correlation between 24-nt siRNAs and CG hypermethylation in the B. cinerea-inoculated samples. In mulberry, many 24-nt siRNAs are derived from TEs, especially from the ends of TEs (Fig. 4b). This may be because long terminal repeats and terminal inverted repeats at both ends of TEs are more likely to form double chains. Among all the TEs, MITE was the main source of 24-nt siRNAs, further demonstrating the importance of MITEs in regulating gene expression through the RdDM pathway.

Promoter methylation is negatively correlated with gene expression levels $^{37,45-47}$, but the correlation between gene body methylation and gene expression is less clear. Our results suggest that gene body methylation may be consistent with promoter region methylation, which is negatively correlated with gene expression (Fig. 6d). DNA hypomethylation of the promoters of nucleotide-binding leucine-rich repeat receptors enhances their expression levels, which subsequently improves resistance. For example, the promoter region of Xa21G was found to be hypomethylated in rice 'Line-2' and hypermethylated in wild-type rice. 'Line-2' showed high levels of gene expression and a resistant phenotype ${ }^{48}$. In $B$. cinereainoculated mulberry samples, two resistance-related genes, Morus017734 and Morus025913, were hypomethylated in the gene body and promoter region, respectively, and their expression levels were significantly upregulated (Fig. 7). Treatment with 5-azacytidine significantly reduced the methylation levels of these two genes, which enhanced their expression and increased mulberry resistance to B. cinerea. In Arabidopsis, mutations in Argonaute 4 increase sensitivity to Pseudomonas syringae, whereas an Arabidopsis mutant deficient in maintaining mCG (met1 mutant) showed enhanced resistance to $P$. syringae ${ }^{5,49}$. In mulberry, resistance to $B$. cinerea was increased by silencing MnMET1 but not by silencing MnAGO4 (Fig. 8). This occurred because MET1 maintains the CG methylation of resistance genes. In conclusion, our whole-genome DNA methylation analysis provided unprecedented insights into the changes in DNA methylation during the interaction between $B$. cinerea and mulberry. These changes affect a large number of proteincoding genes and their transcriptional regulation. Our results also showed that hypomethylation enhanced resistance to $B$. cinerea by inducing the expression of resistance genes. This is useful information for the methylation-targeted breeding of resistant varieties.

\section{Materials and methods}

\section{Plant materials and the $B$. cinerea treatment}

In total, 12 10-month-old mulberry (M. notabilis C.K. Schneid) seedlings were randomly divided into three replicates of two treatments as follows: inoculation with agar blocks without $B$. cinerea (mock-treated; mock) and inoculation with agar blocks containing $B$. cinerea (inoculated). The treated leaves on each plant were in the same position. Three days after the treatment, each 
whole-treated mulberry leaf was removed and sequenced. B. cinerea (MM1 isolate) was isolated from the mature mulberry fruit.

Five-month-old mulberry seedlings were treated with $20 \mathrm{mM}$ of the DNA methylation inhibitor 5-azacytidine (Sigma, St. Louis, MO, USA) dissolved in $\mathrm{ddH}_{2} \mathrm{O}$ with Triton X-100 (0.01\%). The inhibitor was applied to the lower surfaces of leaves. $\mathrm{ddH}_{2} 0$ with Triton X-100 (0.01\%) was also applied to the lower surfaces of leaves in the control group. The treated leaves were wrapped with plastic wrap to prevent the solution from evaporating. This treatment was carried out on 10 June and again on 13 June, and the samples were inoculated with $B$. cinerea on 15 June.

\section{Genome-wide bisulfite sequencing and data analysis}

Genomic DNA was extracted from leaves using the DNeasy plant Maxi Kit (Qiagen, Hilden, Germany). The samples were sequenced at the Beijing Genomics Institute (Shenzhen, China). The construction and sequencing of the bisulfite-seq library were performed as described previously ${ }^{31,50}$. The DNA was fragmented by sonication using a Bioruptor (Diagenode, Seraing, Belgium) into 250bp fragments, on average; then, $\mathrm{dA}$ was added to the $3^{\prime}$ end and ligated with methylated adaptors. The EZ DNA Methylation-Gold kit (Zymo, Orange, CA, USA) was used to convert the ligated DNA. The fragments were sequenced using the Illumina HiSeq4000 platform (Illumina, San Diego, CA, USA). The data were mapped to the mulberry genome using BSMAP ${ }^{51}$. The parameters were set to -d ref.fa -u $-\mathrm{v} 9$-z 33 -p 8 -n 0 -w 20 -s 16 -f 10 $-\mathrm{L} 100$.

The methylation level was determined by dividing the number of reads covering each $\mathrm{mC}$ by the total number of reads ( $\geq 4$ reads effectively) covering that cytosine, which was also equal to the $\mathrm{mC} / \mathrm{C}$ ratio at each reference cyto$\sin ^{52,53}$. The different methylated regions from different groups were calculated using metilene $(\mathrm{v} 0.2-7)^{54}$. The parameters were set to -M $200-\mathrm{m} 5$-d 0.1 -t 1 -f 1 -X 2 -Y $2-\mathrm{v} 0.7$. The significant regions were selected if they met the following criteria: mean methylation difference $\geq 0.1$ and $p$-value $\leq 0.05$. Gene element annotations of the methylated regions or DMRs from the different samples were performed. The GO (http://www.geneontology.org/) analyses and KEGG (https://www.kegg.jp/) enrichments of the DMR-related genes were also determined using phyper (https://en.wikipedia.org/wiki/Hypergeometric_d istribution) to calculate $\mathrm{p}$-values and determine the false discovery rate as assessed using the Bonferroni method. The details of the DMRs are listed in Table S2.

\section{RNA sequencing and data analyses}

Ethanol precipitation and the CTAB-pBIOZOL reagent (Bioer, Hangzhou, China) were used to purify total RNA from plant tissues in accordance with the manufacturer's instructions. The mRNA was purified using magnetic beads with attached oligo (dT)s. First-strand cDNA was generated by random hexamer-primed reverse transcription followed by second-strand cDNA synthesis. For RNA-seq, libraries were constructed and sequenced at the Beijing Genomics Institute using the BGIseq500 platform. The clean reads were mapped to the reference genome using HISAT2 (v2.0.4), and then the expression levels of genes were calculated using RSEM (v1.2.12). A differential expression analysis was performed using DESeq2 (v1.4.5) with a $Q$-value $\leq 0.05$.

\section{Small-RNA sequencing and data analysis}

TRIzol reagent (Ambion, Foster City, CA, USA) was used to extract total RNA from leaves. Small RNAs ( 18-30 nt) were then isolated on denaturing polyacrylamide gels. The libraries were constructed and sequenced at the Beijing Genomics Institute using the BGIseq500 platform. Reads with $18 \mathrm{bp}>$ lengths $>30 \mathrm{bp}$ were discarded. The clean small RNA sequences were mapped to the genome using the anchor alignment-based small RNA annotation (AASRA) program. The mapped reads were normalized to the total clean reads for further analyses.

\section{Identification and phylogenetic analysis of DNA methyltransferases, DNA demethylases, and genes in the RdDM pathway of mulberry \\ BLASTn and BLASTp were performed with Arabidopsis} and strawberry orthologs as queries against the mulberry genome database (https://morus.swu.edu.cn/morusdb/). The strawberry genome (https://phytozome.jgi.doe.gov/ pz/portal.html\#!info?alias=Org_Fvesca) and the Arabidopsis genome (https://www.arabidopsis.org) were used. The full-length cDNA sequences were aligned using ClustalW. The neighbor joining phylogenetic tree was constructed using MEGA6 ${ }^{55}$.

\section{qRT-PCR analysis}

qRT-PCR was performed using an Applied Biosystems StepOnePlus ${ }^{\mathrm{TM}}$ real-time PCR machine with the method described previously ${ }^{20}$. MnActin was used as the internal control for mulberry. Details about the qRT-PCR primers are listed in Table S3.

\section{McrBC-PCR analysis}

McrBC-PCR detected the DNA methylation level within a target range. McrBC digestion was performed with 100ng genomic DNA using the McrBC kit (NEB, Beijing, China) in accordance with the manufacturer's instructions. The negative control was the digestion system without GTP. The primers were designed using Primer5 and are listed in Table S4. 


\section{VIGS and transient overexpression}

To investigate gene functions, VIGS based on TbCSV was used ${ }^{56}$. Fragments of MnMET1 (428 bp), MnAGO4 (419), and MnPDS (188 bp) were PCR-amplified from mulberry cDNA and cloned independently into the 2mDNA1 vector to generate the plasmids 2mDNA1MnMET1, 2mDNA1-MnAGO4, and 2mDNA1-MnPDS, respectively. Mulberry mosaic dwarf-associated virus (MMDaV) was the auxiliary plasmid ${ }^{57}$. MMDaV, 2mDNA1, 2mDNA1-MnMET1, 2mDNA1-MnAGO4, and 2mDNA1-MnPDS were introduced independently into Agrobacterium tumefaciens strain GV3101. MMDaV and 2 mDNA1 or their derivatives were mixed at 1:1 $(\mathrm{v} / \mathrm{v})$ ratios and infiltrated into the cotyledons of 1-week-old mulberry seedlings using a $1-\mathrm{cm}^{3}$ syringe without a needle. The final optical density at a wavelength of $600 \mathrm{~nm}$ of each mixed Agrobacterium culture was 0.5. After $24 \mathrm{~h}$ in the dark, the mulberry seedlings were transferred to a greenhouse. At 14 days post agroinfiltration, the second true leaf was analyzed by qRT-PCR, and the first true leaf was inoculated with $B$. cinerea.

The full-length MnMET1 coding sequence was cloned into the pLGNL vector between the KpnI and EcoRI sites. Then, pLGNL and pLGNL-MnMET1 were introduced independently into A. tumefaciens strain GV3101, which was then infiltrated into the leaves of 1-month-old mulberry seedlings. The primers used are listed in Table S5.

\section{Statistical analyses}

All the graphs represent the results of multiple independent experiments $(n \geq 3)$, and the values are the means with standard deviations (SDs). All the data were analyzed using Excel 2013 (Microsoft, Seattle, WA, USA). A statistical significance analysis was performed using the twotailed unpaired Student's $t$-test, and a $P$-value $<0.05$ was considered to indicate a statistically significant difference.

\section{Acknowledgements}

This project was funded by the National Key Research and Development Program (No. 2018YFD1000602), the Chongqing Research Program of Basic Research and Frontier Technology (cstc2018jcyjAX0407), and the Natural Science Foundation of China (No. 31572323).

\section{Author contributions}

Y.X. and N.H. conceived and designed the research. Y.X. conducted the experiments. Y.X. and B.M. analyzed the data. Q.Z., W.H., and M.Q. provided technical assistance to $Y . X$. in the transgenic experiments. Y.X. wrote the paper, and N.H. revised the paper. All the authors read and approved the paper.

\section{Conflict of interest}

The authors declare no competing interests.

Supplementary information The online version contains supplementary material available at https://doi.org/10.1038/s41438-021-00588-X.
Received: 14 October 2020 Revised: 24 March 2021 Accepted: 19 April 2021 Published online: 01 July 2021

\section{References}

1. Dean, R. et al. The Top 10 fungal pathogens in molecular plant pathology. Mol. Plant Pathol. 13, 414-430 (2012)

2. Legard, D. E., Xiao, C. L., Mertely, J. C. \& Chandler, C. K. Effects of plant spacing and cultivar on incidence of Botrytis fruit rot in annual strawberry. Plant Dis. 84, 531-538 (2000).

3. Angelini, R. M. D. et al. Occurrence of fungicide resistance in populations of Botryotinia fuckeliana (Botrytis cinerea) on table grape and strawberry in southern Italy. Pest Manag. Sci. 70, 1785-1796 (2014).

4. Lamichhane, J. R., Dachbrodt-Saaydeh, S., Kudsk, P. \& Messean, A. Toward a reduced reliance on conventional pesticides in European agriculture. Plant Dis. 100, 10-24 (2016)

5. Dowen, R. H. et al. Widespread dynamic DNA methylation in response to biotic stress. Proc. Natl Acad. Sci. USA 109, E2183-E2191 (2012).

6. Hewezi, T., Howe, P., Maier, T. R. \& Baum, T. J. Arabidopsis small RNAs and their targets during cyst nematode parasitism. Mol. Plant Microbe 21, 1622-1634 (2008).

7. Boyko, A. et al. Transgenerational changes in the genome stability and methylation in pathogen-infected plants (virus-induced plant genome instability). Nucleic Acids Res. 35, 1714-1725 (2007).

8. Yang, S. M., Tang, F., Caixeta, E. T. \& Zhu, H. Y. Epigenetic regulation of a powdery mildew resistance gene in medicago truncatula. Mol. Plant $\mathbf{6}$, 2000-2003 (2013)

9. Zhu, J. K. Active DNA demethylation mediated by DNA glycosylases. Annu. Rev. Genet. 43, 143-166 (2009)

10. Law, J. A. \& Jacobsen, S. E. Establishing, maintaining and modifying DNA methylation patterns in plants and animals. Nat. Rev. Genet. 11, 204-220 (2010).

11. Zemach, A. et al. The Arabidopsis nucleosome remodeler DDM1 allows DNA methyltransferases to access H1-containing heterochromatin. Cell 153, 193-205 (2013).

12. Matzke, M. A., Kanno, T. \& Matzke, A. J. RNA-Directed DNA methylation: the evolution of a complex epigenetic pathway in flowering plants. Annu. Rev. Plant Biol. 66, 243-267 (2015).

13. Matzke, M. A. \& Mosher, R. A. RNA-directed DNA methylation: an epigenetic pathway of increasing complexity. Nat. Rev. Genet. 15, 394-408 (2014).

14. Liu, R. \& Lang, Z. B. The mechanism and function of active DNA demethylation in plants. J. Integr. Plant Biol. 62, 148-159 (2020).

15. Zhang, $\mathrm{H}$. et al. Transposon-derived small RNA is responsible for modified function of WRKY45 locus. Nat. Plants 2, 16016 (2016).

16. Yoder, J. A., Walsh, C. P. \& Bestor, T. H. Cytosine methylation and the ecology of intragenomic parasites. Trends Genet. 13, 335-340 (1997).

17. Deng, Y. et al. Epigenetic regulation of antagonistic receptors confers rice blast resistance with yield balance. Science 355, 962-965 (2017).

18. Tirnaz, S. \& Batley, J. DNA methylation: toward crop disease resistance improvement. Trends Plant Sci. 24, 1137-1150 (2019).

19. Jiang, Y. \& Nie, W. J. Chemical properties in fruits of mulberry species from the Xinjiang province of China. Food Chem. 174, 460-466 (2015).

20. Xin, Y. C., Meng, S., Ma, B., He, W. M. \& He, N. J. Mulberry genes MnANR and MnLAR confer transgenic plants with resistance to Botrytis cinerea. Plant Sci. 296, 110473 (2020)

21. He, N. et al. Draft genome sequence of the mulberry tree Morus notabilis. Nat. Commun. 4, 2445 (2013)

22. Ma, B., Li, T., Xiang, Z. \& He, N. MnTEdb, a collective resource for mulberry transposable elements. Database 2015, bav004 (2015).

23. Xin, Y. C., Ma, B., Xiang, Z. H. \& He, N. J. Amplification of miniature invertedrepeat transposable elements and the associated impact on gene regulation and alternative splicing in mulberry (Morus notabilis). Mobile DNA 10, 27 (2019).

24. Song, Q. X. et al. Genome-wide analysis of DNA methylation in soybean. Mol. Plant 6, 1961-1974 (2013).

25. Zakrzewski, F., Schmidt, M., Van Lijsebettens, M. \& Schmidt, T. DNA methylation of retrotransposons, DNA transposons and genes in sugar beet (Beta vulgaris L.). Plant J. 90, 1156-1175 (2017).

26. Huang, $\mathrm{H}$. et al. Global increase in DNA methylation during orange fruit development and ripening. Proc. Natl Acad. Sci. USA 116, 1430-1436 (2019). 
27. Cheng, J. et al. Downregulation of RdDM during strawberry fruit ripening. Genome Biol. 19, 212 (2018).

28. Cokus, S. J. et al. Shotgun bisulphite sequencing of the Arabidopsis genome reveals DNA methylation patterning. Nature 452, 215-219 (2008).

29. Zhang, J. et al. Autotetraploid rice methylome analysis reveals methylation variation of transposable elements and their effects on gene expression. Proc. Natl Acad. Sci. USA 112, E7022-E7029 (2015).

30. Zhong, S. et al. Single-base resolution methylomes of tomato fruit development reveal epigenome modifications associated with ripening. Nat. Biotechnol. 31, 154-159 (2013).

31. $\mathrm{Xu}, \mathrm{J}$. et al. Single-base methylome analysis reveals dynamic epigenomic differences associated with water deficit in apple. Plant Biotechnol. J. 16, 672-687 (2018).

32. Liang, D. et al. Single-base-resolution methylomes of Populus trichocarpa reveal the association between DNA methylation and drought stress. BMC Genet. 15, S9 (2014).

33. Ausin, l. et al. DNA methylome of the 20-gigabase Norway spruce genome. Proc. Natl Acad. Sci. USA 113, E8106-E8113 (2016).

34. Niederhuth, C. E. et al. Widespread natural variation of DNA methylation within angiosperms. Genome Biol. 17, 194 (2016)

35. Hsieh, T. F. et al. Genome-wide demethylation of Arabidopsis endosperm. Science 324, 1451-1454 (2009).

36. Zemach, A. et al. Local DNA hypomethylation activates genes in rice endosperm. Proc. Natl Acad. Sci. USA 107, 18729-18734 (2010).

37. Singer, T., Yordan, C. \& Martienssen, R. A. Robertson's mutator transposons in A. thaliana are regulated by the chromatin-remodeling gene decrease in DNA methylation (DDM1). Genes Dev. 15, 591-602 (2001).

38. Biemont, C. \& Vieira, C. Genetics: junk DNA as an evolutionary force. Nature 443, 521-524 (2006)

39. Wang, C. et al. A transposon-directed epigenetic change in ZmCCT underlies quantitative resistance to Gibberella stalk rot in maize. New Phytol. 215, 1503-1515 (2017).

40. Schumann, U. et al. DEMETER plays a role in DNA demethylation and disease response in somatic tissues of Arabidopsis. Epigenetics 14, 1074-1087 (2019).

41. Schumann, U., Lee, J., Kazan, K., Ayliffe, M. \& Wang, M. B. DNA-demethylase regulated genes show methylation-independent spatiotemporal expression patterns. Front. Plant Sci. 8, 1449 (2017).
42. Le, T. N. et al. DNA demethylases target promoter transposable elements to positively regulate stress responsive genes in Arabidopsis. Genome Biol. 15, 458 (2014).

43. Cao, X. \& Jacobsen, S. E. Role of the arabidopsis DRM methyltransferases in de novo DNA methylation and gene silencing. Curr. Biol. 12, 1138-1144 (2002).

44. Mosher, R. A. \& Melnyk, C. W. siRNAs and DNA methylation: seedy epigenetics. Trends Plant Sci. 15, 204-210 (2010)

45. Wang, $\mathrm{H}$. et al. CG gene body DNA methylation changes and evolution of duplicated genes in cassava. Proc. Natl Acad. Sci. USA 112, 13729-13734 (2015).

46. Zhang, $X$. et al. Genome-wide high-resolution mapping and functional analysis of DNA methylation in arabidopsis. Cell 126, 1189-1201 (2006).

47. Jones, P. A. Functions of DNA methylation: islands, start sites, gene bodies and beyond. Nat. Rev. Genet. 13, 484-492 (2012).

48. Akimoto, K. et al. Epigenetic inheritance in rice plants. Ann. Bot. 100, 205-217 (2007).

49. Agorio, A. \& Vera, P. ARGONAUTE4 is required for resistance to Pseudomonas syringae in Arabidopsis. Plant Cell 19, 3778-3790 (2007).

50. Lu, X. et al. Single-base resolution methylomes of upland cotton (Gossypium hirsutum L.) reveal epigenome modifications in response to drought stress. BMC Genomics 18, 297 (2017).

51. Xi, Y. \& Li, W. BSMAP: whole genome bisulfite sequence MAPping program. BMC Bioinform. 10, 232 (2009).

52. Lister, R. et al. Human DNA methylomes at base resolution show widespread epigenomic differences. Nature 462, 315-322 (2009).

53. Xiang, $\mathrm{H}$. et al. Single base-resolution methylome of the silkworm reveals a sparse epigenomic map. Nat. Biotechnol. 28, 516-520 (2010).

54. Juhling, F. et al. metilene: fast and sensitive calling of differentially methylated regions from bisulfite sequencing data. Genome Res. 26, 256-262 (2016).

55. Tamura, K., Stecher, G., Peterson, D., Filipski, A. \& Kumar, S. MEGA6: molecular evolutionary genetics analysis version 6.0. Mol. Biol. Evol. 30, 2725-2729 (2013).

56. Huang, C., Xie, Y. \& Zhou, X. Efficient virus-induced gene silencing in plants using a modified geminivirus DNA1 component. Plant Biotechnol. J. 7, 254-265 (2009).

57. Ma, Y. et al. Identification and molecular characterization of a novel monopartite geminivirus associated with mulberry mosaic dwarf disease. J. Gen. Virol. 96, 2421-2434 (2015). 